\title{
STD/HIV control in Malawi and the search for affordable and effective urethritis therapy: a first field evaluation
}

G Lule, F M-T Behets, I F Hoffman, G Dallabetta, H A Hamilton, S Moeng, G Liomba, M S Cohen

\begin{abstract}
Objectives-To evaluate gonococcal (GU) and nongonococcal urethritis (NGU), chlamydia antigen, and serostatus for syphilis and human immunodeficiency virus (HIV) among males attending a Malawian STD clinic with complaints of urethral discharge and/or dysuria. To collect demographic and behavioural data and to determine the effectiveness of five treatments for urethritis.

Methods-Urethritis was diagnosed using microscopy and culture for Neisseria gonorrhoeae. Sera were screened with rapid plasma reagin ( $R P R)$ and if reactive, with microhaemagglutination for Treponema pallidum (MHA-TP). HIV antibodies and chlamydia antigen were detected using enzyme immunoassay. Patients were randomised for treatment, cure was assessed 8-10 days later.

Results-At enrolment, GU was diagnosed in $415(80 \cdot 3 \%)$ and NGU in 59 $(11.2 \%)$ of 517 males. Chlamydia antigen was found in $26(5 \cdot 2 \%)$ of 497 specimens tested. Syphilis seropositivity rate (RPR and MHA-TP reactive) was $10 \cdot 7 \%$. Overall HIV seroprevalence was $44 \cdot 2 \%$; $71.7 \%$ of men with reactive syphilis serology were HIV $(+)$ compared with $40.9 \%$ of syphilis seronegatives (OR: 3.6 , p $<0.001)$. Trimethoprim $320 \mathrm{mg} /$ sulphamethoxazole $1600 \mathrm{mg}$ by mouth for 2 days (TMPSMX), or the combination of amoxicillin $3 \mathrm{gm}$, probenicid $1 \mathrm{gm}$, and clavulanate $125 \mathrm{mg}$ by mouth once (APC), failed to cure gonorrhoea effectively. Amoxicillin $3 \mathrm{gm}$, probenicid 1 gm, and clavulanate $125 \mathrm{mg}$, by mouth once with doxycycline $100 \mathrm{mg}$ BID for 7 days (APC-D), gentamicin $240 \mathrm{mg}$ IM once (GENT), ciprofloxacin $250 \mathrm{mg}$ by mouth once (CIPRO) cured $92.9 \%$ to $95 \%$ of gonorrhoea. APC-D treatment did not generate less NGU at follow-up. HIV serostatus did not affect cure of urethritis.

Conclusion-All patients presenting with urethritis should be treated syndromically using a simple algorithm and screened for syphilis seroreactivity for appropriate treatment and counselling.
\end{abstract}

(Genitourin Med 1994;70:384-388)
Introduction

The World Health Organisation's (WHO) Global Program on AIDS (GPA) estimated by mid-1993 that of the 13 million cumulative HIV infections in adults and youth worldwide, more than 8 million occurred in Africa. ${ }^{1}$ Numerous cross-sectional and prospective studies provide consistent evidence that nonulcerative as well as ulcerative sexually transmitted diseases (STDs) are associated with a 3- to 5-fold increased risk of HIV transmission. ${ }^{2}$ Although the relative risk for transmission of HIV infection might be higher for ulcerative diseases, in many settings the attributable risk associated with gonococcal and chlamydial infections will be more consequential. ${ }^{2}$ Indeed, according to WHO estimates for $1991,29.9 \%$ of 250.5 million new cases of classic STDs (that is, all STDs except HIV) worldwide were due to gonococcal and chlamydial infections compared with $2 \cdot 2 \%$ for syphilis and chancroid. ${ }^{3}$ Adequate control of these infections has become an essential component of most HIV prevention programmes. ${ }^{4}$

Malawi has high HIV infection rates. At the Blantyre Queen Elisabeth Central Hospital (QECH), HIV-1 seropositivity was $23 \%$ among 6,600 antenatal women in $1989-1990,{ }^{5}$ and between $15 \%$ and $30 \%$ among blood donors in $1988 .{ }^{6}$ In Lilongwe, $62.4 \%$ of 705 adult outpatients with signs or symptoms associated with STDs had HIV antibodies in $1989 .^{6}$

Sexually transmitted infections other than HIV are widespread in Malawi, although less well documented. 6 A $4.4 \%$ prevalence of STDs was found among adult outpatients presenting at the Kamuzu Central Hospital in Lilongwe, Malawi. This underestimates the true prevalence because the study design did not allow detection of asymptomatic STDs, and laboratory diagnosis was limited. ${ }^{6}$ Among the 6,600 women mentioned earlier who were seeking antenatal care in Blantyre, $42 \%$ were diagnosed with a classic STD. ${ }^{5}$

Effective STD control requires proper diagnosis and case management. Syndromic approaches using simple algorithms have been advocated. ${ }^{1}$ Knowledge of local factors such as relative contribution of aetiological agents associated with specific syndromes, effectiveness of antimicrobial therapies, and behavioural observations are keys to optimal design and use of syndromic approaches. During the 
recent launching of its STD control programme, the Malawian AIDS Secretariat in collaboration with other scientists, found that $39 \%$ of the gonococcal isolates produced penicillinase and evaluation of in vitro antimicrobial susceptibility was started (personal communication, Dr Dallabetta).

This study was undertaken to determine the relative contribution of Neisseria gonorrhoeae and Chlamydia trachomatis to urethritis in Malawi, to evaluate the effectiveness of five antibiotic therapies for urethritis, and to determine clinical, demographic and behavioural characteristics of male patients presenting with complaints of urethritis in an urban clinic.

\section{Methods}

The study was approved by the Health Sciences Research Committee of Malawi, by the Committee on the Protection of the Rights of Human Subjects at the University of North Carolina School of Medicine, and by the review board of Family Health International. Antimicrobials previously described as effective and recorded in the Malawian national drug formulary were selected for evaluation together with ciprofloxacin, a well established gonococcal therapy. Between September 1992 and March 1993, all males presenting with complaints of urethral discharge and/or dysuria (even when no urethral discharge was seen upon examination) at the outpatient clinic of the Queen Elisabeth Central Hospital in Blantyre, Malawi, were asked to participate in a prospective study of urethritis treatment efficacy. Only those patients who had no concurrent visible ulcer, and who agreed to return for a follow-up visit were eligible for enrolment in the study. After obtaining informed consent from potential study subjects, a trained nurse administered a structured, nurse-led questionnaire in the local language to collect demographic and behavioral data as well as a selected medical history.

Each participant underwent a physical examination including urethral stripping to determine presence of discharge. Urethral samples were obtained for microscopy of Gram stained smear, for culture of Neisseria gonorrhoeae and for EIA chlamydia antigen detection. Urethral specimens were directly inoculated onto Thayer-Martin media (BBL, Becton-Dickinson, USA) and immediately placed in candle extinction jars with moisture pads. Cultures were incubated at $36^{\circ} \mathrm{C}$ and inspected for growth indicative of Neisseria gonorrhoeae after 24 and 48 hours. A Gram stain was performed on oxidase positive colonies with characteristic morphology, and pure subcultures were frozen for further analyses. A patient was diagnosed with gonococcal urethritis (GU) if Gram negative intracellular diplococci were found in the urethral smear and/or if the culture was positive. Chlamydia antigen detection was carried out using EIA (Antigenz EIA, Shield Diagnostics Ltd, UK) following the instructions of the manufacturer. A patient was diagnosed with nongonococcal urethritis (NGU) when the culture for Neisseria gonorrhoeae remained negative and when at least five polymorphonuclear cells were observed per high power field in the direct urethral smear in the absence of Gram negative intracellular diplococci. Patients who had less than 5 polymorphonuclear cells per high power field, and had no evidence of gonococcal urethritis but had a positive chlamydia antigen detection test were also diagnosed with NGU.

Blood was collected by venipuncture for screening of sera with rapid plasma reagin (RPR) (Macro-Vue, Becton-Dickinson, USA). RPR reactive sera were diluted to determine endpoint reactivity and were tested with microhemagglutination for Treponema pallidum (MHA-TP) (SeraTec Treponemal Antibody, Fujeribio, Miles, USA).

Upon completion of the study, linked, anonymous testing for HIV antibodies using Wellcozyme EIA (Wellcome, UK) was carried out on stored sera. Reactive sera underwent repeat testing.

Patients were randomised using computerised randomisation assignments in sealed envelopes to one of the following treatment arms: 1. Amoxicillin $3 \mathrm{gm}$, probenicid $1 \mathrm{gm}$, and clavulanate $125 \mathrm{mg}$ by mouth once (APC), 2. Amoxicillin $3 \mathrm{gm}$, probenicid $1 \mathrm{gm}$, and clavulanate $125 \mathrm{mg}$, by mouth once and doxycycline $100 \mathrm{mg}$ BID for 7 days (APC-D), 3. Gentamicin $240 \mathrm{mg}$ IM once (GENT), 4. Ciprofloxacin $250 \mathrm{mg}$ by mouth once (CIPRO), 5. Trimethoprim $320 \mathrm{mg} /$ sulphamethoxazole $1600 \mathrm{mg}$ by mouth for 2 days (TMPSMX)

All patients were asked to return 8-10 days after the initial visit for assessment of symptoms and signs. A physical examination was performed as described above to determine presence of discharge, and a specimen for urethral Gram stain and culture was obtained. Chlamydial antigen was evaluated only if the symptoms persisted.

dBASE III PLUS was used for data entry. SAS statistical software for analyses. Comparisons of rates were carried out using Mantel-Haenszel chi-square, Fisher's exact, the nonparametric Wilcoxon test was used for ordinal data.

\section{Results}

A total of 517 male subjects were enrolled in the study. The mean age of the study participants was $27 \cdot 3$ years (range: 13-70, SE: 0.35 ) with an average of 5.4 years (SE: $0 \cdot 19$ ) of education completed. The mean duration of reported symptoms at initial visit was 8.7 days (range: $1-85$, SE: 0.76 ), $12.2 \%$ of the patients reported presence of symptoms for at least two weeks. When asked about care-seeking behaviour for the current episode of urethritis, $53 \%$ of the patients reported having sought treatment elsewhere: $36.5 \%$ had consulted a traditional healer or herbalist, $31.0 \%$ had gone to a pharmacist, $22.7 \%$ to another clinic or private physician, and $29.7 \%$ 
Table 1 Characteristics of male patients with urethritis reporting to $Q E H$, Blantyre, Malawi between September 1992 and March 1993

\begin{tabular}{lr}
\hline & $\%$ \\
\hline Single (never married) & $46 \cdot 2$ \\
Married & $47 \cdot 5$ \\
Divorced/widowed & $6 \cdot 3$ \\
Employed & $70 \cdot 2$ \\
Circumcised & $13 \cdot 6$ \\
History of STD in past 2 years & $22 \cdot 3$ \\
History of STD in past 6 months & $8 \cdot 9$ \\
> 1 sex partner in past month & 13.9 \\
Sex with bargirl in past month & $64 \cdot 4$ \\
Sex with a new partner in past month & 3.9 \\
\hline
\end{tabular}

reported other unspecified forms of treatment. Some patients thus reported having sought more than one form of treatment. Additional behavioural and demographic characteristics of the study participants are described in table 1 . Reported sexual contact with a bargirl (commercial sex worker) did not correlate with employment or marital status.

Purulent discharge was observed among $71 \cdot 4 \%$ of the patients, $27 \cdot 2 \%$ had mucoid discharge, and $1.3 \%$ had no visible secretion.

Table 2 (a) Cure rates of urethritis according to symptoms reported by patients at followup visit

\begin{tabular}{|c|c|c|c|c|}
\hline Therapy $(*)$ & $\begin{array}{l}\text { Number of } \\
\text { patients }\end{array}$ & $\begin{array}{l}\text { \% No } \\
\text { symptoms }\end{array}$ & $\begin{array}{l}\text { \% Decreased } \\
\text { symptoms }\end{array}$ & $\begin{array}{l}\text { \% Samel } \\
\text { worse }\end{array}$ \\
\hline $\begin{array}{l}\text { APC } \\
\text { APC-D } \\
\text { GENT } \\
\text { CIPRO } \\
\text { TMPSMX } \\
\text { Total }\end{array}$ & $\begin{array}{l}76 \\
74 \\
60 \\
74 \\
41 \\
325\end{array}$ & $\begin{array}{l}61 \cdot 8 \\
78 \cdot 4 \\
66 \cdot 7 \\
77 \cdot 0 \\
53 \cdot 7 \\
68 \cdot 9\end{array}$ & $\begin{array}{l}29 \cdot 0 \\
12 \cdot 2 \\
25 \cdot 0 \\
10 \cdot 8 \\
31 \cdot 7 \\
20 \cdot 6\end{array}$ & $\begin{array}{r}9 \cdot 2 \\
9 \cdot 5 \\
8 \cdot 3 \\
12 \cdot 2 \\
14 \cdot 6 \\
10 \cdot 5\end{array}$ \\
\hline
\end{tabular}

(b) Cure rates of urethritis according to occurrence of laboratory confirmed urethritis at follow-up visit

\begin{tabular}{|c|c|c|c|c|}
\hline Therapy $\left(^{*}\right)$ & $\begin{array}{l}\text { Number of } \\
\text { patients }\end{array}$ & $\%$ Cured & $\begin{array}{l}\% N G U I \\
P G U\left(^{*}\right)\end{array}$ & $\% G U\left({ }^{*}\right)$ \\
\hline $\begin{array}{l}\text { APC } \\
\text { APC-D } \\
\text { GENT } \\
\text { CIPRO } \\
\text { TMPSMX } \\
\text { Overall }\end{array}$ & $\begin{array}{l}71 \\
68 \\
53 \\
70 \\
35 \\
297\end{array}$ & $\begin{array}{l}56 \cdot 3 \\
73 \cdot 5 \\
73 \cdot 6 \\
61 \cdot 4 \\
51 \cdot 4 \\
65 \cdot 6\end{array}$ & $\begin{array}{r}15 \cdot 5 \\
20 \cdot 6 \\
22 \cdot 6 \\
28 \cdot 6 \\
5 \cdot 7 \\
20 \cdot 2\end{array}$ & $\begin{array}{r}28 \cdot 2 \\
5 \cdot 9 \\
3 \cdot 8 \\
10 \cdot 0 \\
42 \cdot 9 \\
14 \cdot 2\end{array}$ \\
\hline
\end{tabular}

${ }^{\star}$ ) See text for abbreviations and definitions.

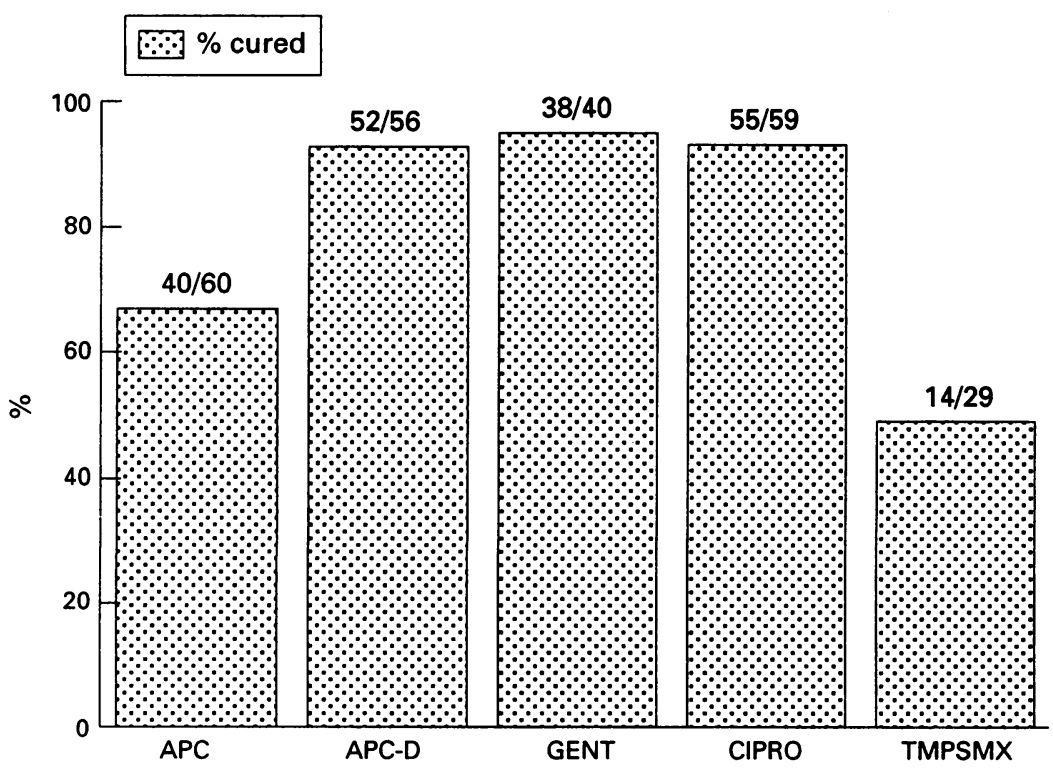

Figure Cure rates of laboratory confirmed gonococcal urethritis.
Scrotal pain was reported by $29 \cdot 1 \%$ of the patients and scrotal swelling by $1 \cdot 8 \%$.

Four hundred and fifteen $(80.3 \%)$ of 517 patients had gonococcal urethritis, and 59 (11.5\%) had nongonococcal urethritis. Chlamydia antigen was found in $26(5 \cdot 2 \%)$ of the 497 specimens tested; the total chlamydia and gonococcal co-infection rate was $2 \cdot 4 \%$. Gonococcal urethritis was diagnosed in $63.1 \%$ of patients with mucoid discharge compared with $86.3 \%$ in patients with purulent secretion (OR: $0 \cdot 27,95 \%$ CI: $0 \cdot 16-0 \cdot 45$; $\mathrm{p}=0.008)$. Sixty one $(12 \cdot 2 \%)$ of 502 evaluated sera were RPR reactive, the overall proportion of RPR and MHA-TP positive sera was $10 \cdot 7 \%$.

Of the 517 patients enrolled in the study, $330(63 \cdot 8 \%)$ returned for their follow-up visit. Men who reported previous use of pills for the current episode of urethritis were less likely to return for follow-up (OR: $0.59 ; 95 \% \mathrm{CI}$ : $0.40-0.85 ; p=0.005)$. Age, circumcision, duration of symptoms, sexual contact with prostitute, marital and employment status, and HIV serostatus did not differ between patients who returned and those who were lost to follow-up. There were no differences in follow-up rates between treatment arms. On average, the patients returned after 9.5 days (1-44 days, median 8 days).

The clinical effects of therapy on all urethritis patients are shown in the first part of table 2 . The best results as reported by the patients were obtained with APC-D or CIPRO, followed by GENT and APC. Only $53.7 \%$ of the patients treated with TMPSMX reported resolution of symptoms at the follow-up visit. Treatment with trimethoprim/sulfamethoxazole was stopped before completion of the study when its poor cure rate became obvious during an interim data analysis by an independent observer.

The effects of therapy on all urethritis patients were further analysed based on laboratory data at follow-up as shown in the second part of table 2. Occurrence of NGU/PGU at follow-up was not lower in patients treated with APC-D, the only regimen with doxycycline. Chlamydia antigen was detected in three $(5 \cdot 8 \%)$ of 55 patients presenting with persistent symptoms at follow-up visit.

The effects of therapy on laboratory-proven gonorrhoea are shown in the fig. APC-D, GENT, and CIPRO were effective at treating gonorrhoea. Confirmed gonococcal urethritis was clearly inadequately cured using TMPSMX or using APC. Among those patients who reported complete disappearance of symptoms, $10.6 \%$ had laboratory evidence of gonococcal infection. Indeed, of the 48 patients with persistent gonococcal infection at follow-up visit, $14.6 \%$ of the patients reported same or worse symptoms, $39.6 \%$ reported decreased symptoms, and $45.8 \%$ reported complete dissipation of initial complaints. Among these 48 patients, 6 (12.5\%) reported having had sex between initial and follow-up visit, compared with $21(8.4 \%)$ of 249 men for whom gonococcal infection was not detected at follow-up $(p=0 \cdot 4)$. 
Two hundred and twenty eight $(44 \cdot 2 \%)$ of 516 patients evaluated had HIV antibodies by EIA. Men between 25-29 years old had the highest rate of seropositivity (58.1\%). Employed men were more likely to be seropositive than unemployed, with HIV prevalence rates of $47.5 \%$ and $36.0 \%$ respectively (OR: $1 \cdot 6,95 \%$ CI: $1 \cdot 09-2 \cdot 38$; $\mathrm{p}=0.02)$. Men reporting alcohol use showed a higher HIV rate: $55 \cdot 2 \%$, than men who did not report alcohol use: $31 \cdot 7 \%$ (OR: $2 \cdot 6$, 95\% CI: $1.85-3.80 ; \mathrm{p}<0.001)$. Although reported sexual contact with a bargirl and lack of circumcision were associated with a higher HIV seroprevalence, these differences were not statistically significant at the 0.05 level. The highest HIV seroprevalence was found among patients with a positive syphilis serology: $71.7 \%$ as compared with $40.9 \%$ among patients with negative syphilis serology (OR: $3 \cdot 6$, 95\% CI: $2 \cdot 01-6 \cdot 65$; $p<0 \cdot 001)$. Cure rates defined by absence of laboratory confirmed urethritis at follow-up visit did not differ significantly between HIV seropositive $(64.2 \%)$ and HIV seronegative patients $(65 \%)$. Similarly, clearance of symptoms as reported by patients at follow-up did not differ significantly between HIV seropositive and HIV seronegative patients.

\section{Discussion}

In this study, infection with Neisseria gonorrhoeae was confirmed in $80.3 \%$ of all patients presenting with complaints of urethral discharge and/or dysuria, whereas Chlamydia trachomatis (as determined by direct antigen detection using EIA) was not a major pathogen. A similarly low prevalence of chlamydia $(3 \cdot 4-4 \%)$ was found among pregnant women in Blantyre. ${ }^{5}$ Among patients symptomatic at follow-up visit we also detected few carriers of chlamydia antigen. We did not screen for other organisms associated with NGU such as Ureaplasma urealyticum and Trichomonas vaginalis.

At enrolment, complaints of dysuria and/or urethral discharge among our study participants were highly specific for laboratory confirmed urethritis. In this setting, the syndromic approach for management of patients presenting with complaints of dysuria and/or urethral discharge using a simple algorithm requires no laboratory analyses except syphilis screening. Our findings are not applicable for asymptomatic patients who do require laboratory diagnosis for detection. For instance, in a community-based study in Tanzania, asymptomatic infection was found in $90 \%$ of men infected with gonorrhoea or chlamydia. ${ }^{7}$ The importance of using effective therapy was illustrated at follow-up where we documented that $85 \%$ of the men who had gonococcal infection reported complete resolution or substantial improvement of symptoms.

Trimethoprim $320 \mathrm{mg} /$ sulphamethoxazole $1600 \mathrm{mg}$ PO for 2 days or amoxicillin $3 \mathrm{gm}$, probenicid $1 \mathrm{gm}$, and clavulanate $125 \mathrm{mg} \mathrm{PO}$ once were ineffective for treatment of gono- coccal urethritis. The failure rates were $51 \cdot 7 \%$ and $33.3 \%$ respectively. Possible reasons for treatment failure such as lack of compliance or vomiting cannot be determined. First doses of all oral treatments were taken under direct supervision of the nurse, but patients' compliance for the second dose of TMPSMX was not directly measured. The three other evaluated regimens: APC-D, GENT, and CIPRO all appear equally effective for gonococcal urethritis with failure rates ranging between $5 \%$ and $7 \cdot 1 \%$.

It is plausible that the urethritis cure rates we observed represent an under-estimate of the real cure rates. Indeed, our treatment failure rates might be higher than we would have observed had all enrolled patients returned for follow-up. Although we did not find significant differences in follow-up rates between treatment arms, patients with prompt response to therapy might be less likely to return for follow-up. In a simultaneously conducted study at the same clinic, we observed an overall follow-up rate among patients with genital ulcer disease (which usually needs more time to cure than urethritis) of $70.2 \%$, compared with $63.8 \%$ among patients with urethral discharge $(p=0.02)$ (manuscript in preparation).

The only doxycyclin-inclusive regimen, APC-D, failed to show a lower NGU rate at follow-up than the other regimens although it reduced urethral symptoms best according to patient reporting. Poor response of Chlamydia trachomatis-negative NGU to antimicrobials which are active against $C$ trachomatis has been described. ${ }^{8}$

Over and Piot have demonstrated that STD control interventions can be highly costeffective. ${ }^{9}$ They calculated that the cost per discounted disability-adjusted life-year saved can be as low as US $\$ 0.56$ for a classic STD control programme aimed at "core groups" or high frequency transmitters. While cost-efficiency calculations provide the rationale for investment in STD control, many developing countries suffer from severe scarcity of resources at the national and individual level. Malawi's per capita GNP of US $\$ 230$, and per capita total health expenditure of US $\$ 11^{10}$ mandate effective STD therapy at the lowest cost possible. Antimicrobial regimens currently recommended by the World Health Organization for treatment of gonorrhoea such as ciprofloxacin $500 \mathrm{mg}$ or ceftriaxone $250 \mathrm{mg}$, are expensive. In the United States Public Health Services, ceftriaxone 125 $\mathrm{mg}$ intramuscularly is recommended for treatment of uncomplicated gonorrhoea. ${ }^{11}$ Even this reduced treatment format remains costly and might be incompatible with many national health budgets. Following completion of this study, the Malawian STD Advisory Committee decided to select gentamicin $240 \mathrm{mg}$ IM once together with doxycycline $100 \mathrm{mg}$ BID for 7 days as the nationally recommended regimen of choice for urethritis. While the results of this study do not necessarily support the use of doxycycline, concurrent use of gentamycin and 
doxycycline might help delay the emergence of gonococcal resistance to gentamycin. The disadvantages and risks associated with injection-requiring gentamycin and the need for sterile needles and syringes were weighed against the local cost of this regimen at US $\$ 0.77$ compared with US $\$ 1.5$ to 2.2 for the other identified effective regimens.

As expected, HIV seroprevalence was high among these patients. Higher socio-economic status as measured by husband's education, was a risk factor for HIV infection among pregnant women in Blantyre. ${ }^{5}$ Among our STD patients we found that employed men and men reporting current alcohol use were at increased risk of being HIV seropositive. We also documented that more than two out of three of the male urethritis patients who had a positive syphilis serology were HIV seropositive. Systematic syphilis screening of all urethritis patients is thus crucial for appropriate treatment including education and counselling. Although our sample size does not allow definitive conclusions, the finding that cure of uncomplicated gonorrhoea was not affected by concurrent HIV infection is encouraging.

In this study, $53 \%$ of all participants presented at the public STD clinic (where all services and drugs are provided free of cost) had first sought care elsewhere for their episode of urethritis. Although we can only speculate about the reasons for this, it does demonstrate the frequency of inadequate care at the first visit. Among our study participants who had sought care elsewhere, more than one out of three reported having consulted a traditional healer or herbalist. Traditional healers have been reported to be perceived as less expensive, more sympathetic, more confidential, and more easily accessible. ${ }^{12}$ That adequate care can increase clinic use without special promotional or educational efforts has been confirmed at this STD clinic in Blantyre where attendance has tripled (personal observation, G. Lule) since an effective regimen is given routinely.

We thank Mr Joe Canner for assistance with data entry and management, Ms Anne-Marie Wangel for clinical and organisational help, Drs Celine Costello Daly and P Frederick Sparling for reviewing the manuscript. The study was funded by the United States Agency for International Development.

1 Merson M. Slowing the spread of HIV: agenda for the 1990s. Science 1993;260:1266-8.

2 Wasserheit JN. Epidemiological synergy: interrelationships between human immunodeficiency virus infection and other sexually transmitted diseases. STD 1992;19: 61-77

3 Khanna J, Van Look PF, Griffin PD. Reproductive health: a key to a brighter future: biennial report 1990-1991. Geneva, World Health Organisation, 1992. $171 \mathrm{p}$ 4 Cohen MS, Dallabetta G, Laga $M$, Holmes KK. A new
deal in HIV prevention: lessons from the global approach. Ann Int Med 1994;120:340-1.

5 Dallabetta G, Miotti PG, Chipangwi JD, et al. High socioeconomic status is a risk factor for human immunodeficiency virus type 1 (HIV-1) infection but not for sexually transmitted diseases in women in Malawi: implications for HIV-1 control. F Infect Dis 1993;167: 36-42.

6 Kristensen JK: The prevalence of symptomatic sexually transmitted diseases and human immunodeficiency virus in outpatients in Lilongwe, Malawi. Genitourin Med in outpatients

7 Grosskurth H, Plummer F, Mhalu F, Mabey D. STD research in Africa. Lancet 1993;342:1415-6.

8 Bowie WR: Urethritis in males. Holmes KK, Mardh P-A Sparling PF, et al. eds. Sexually Transmitted Diseases. 1993 New York. Oxford University Press.

9 Over Mead, Piot Peter. HIV Infection and sexually transmitted diseases. In Jamison DT, Mosley WH, Measham AR, Bobadilla JL, eds. Disease Control Priorities in Developing Countries. 1993, Washington, DC, USA.

10 The World Bank: World Development Report 1993. Oxford Oxford University Press.

11 Centers for Disease Control and Prevention. 1993 Sexually transmitted diseases treatment guidelines. MMWR 1993;42:57.

12 Dallabetta G, Allen H, Helitzer-Allen D, et al. Sexually transmitted infections (STI) in Malawi: local perceptransmitted infections (STI) in Malawi: local percepConference in AIDS, 1993, Berlin, Germany 